\title{
A Survey of Articles in the Newsletter of the EMS about the History and Activities of Full Member Societies of the EMS (Issue 1, September 1991 - Issue 104, June 2017)
}

Fernando Pestana da Costa (Universidade Aberta, Lisboa, Portugal), Editor of the EMS Newsletter

The architecture of the European Mathematical Society allows for individual and corporate members; in the latter category, there is a sub-category of "full members" that is made up of mathematical societies of the various countries of the European continent (plus Israel).

Full member societies have had a presence in the Newsletter of the EMS since its very first issue, with information articles about their history and activities. These are not just short announcements of their current initiatives (which are also regularly provided) but also short papers (typically 1-3 pages long) describing something of their history as well as their most important past and current activities.

Unfortunately, in spite of the existence of open access to all Newsletter issues on the webpage of the EMS Pub- lishing House (http://www.ems-ph.org/journals/all_issues. php?issn=1027-488X), it is not exactly easy to check for published articles about EMS full members. In order to have a clearer idea of what has been published and so to be able to plan future actions, the Editorial Board of the Newsletter has recently compiled a survey of that information. We believe this survey may also be of interest to all EMS members and to the general readership of the Newsletter, as it provides a direct pointer to reliable published information about mathematical societies in Europe that can be difficult to obtain otherwise. In Table 1, we present the results of the survey. As part of Editorial Board duties, in due time and according to the planned Editorial Board policy, societies that have not yet been covered in the Newsletter will be asked to contribute.

Survey $^{12}$ of articles about EMS full member ${ }^{3}$ societies published in the Newsletter up to issue 104

\begin{tabular}{|l|l|l|l|l|}
\hline Name of Society & Country & \multicolumn{2}{l|}{$\begin{array}{l}\text { Founding } \\
\text { Date }\end{array}$} & \multicolumn{2}{l|}{ Published in } \\
\cline { 4 - 5 } & & Issue & Date \\
\hline Armenian Mathematical Union & Armenia & 1901 & & \\
\hline $\begin{array}{l}\text { Association for Mathematics Applied to Economics and } \\
\text { Social Sciences }\end{array}$ & Italy & 1976 & 96 & $06 / 2015$ \\
\hline Association of Mathematicians of Republic of Macedonia & Macedonia & & & \\
\hline Austrian Mathematical Society & Austria & 1903 & & \\
\hline Belarusian Mathematical Society & Belarus & & & \\
\hline Belgian Mathematical Society & Belgium & 1921 & 29 & $09 / 1998$ \\
\hline Bosnian Mathematical Society & & & 80 & $06 / 2011$ \\
\hline Catalan Mathematical Society & Bosnia & & & \\
\hline Croatian Mathematical Society & Spain & 1931 & 93 & $09 / 2014$ \\
\hline Cyprus Mathematical Society & Croatia & & & \\
\hline
\end{tabular}

\footnotetext{
1 Information about the founding date of some societies could not be obtained from the data in the EMS webpage and archives. We thank Elvira Hyvönen, secretary of the EMS, her help in procuring this information.

2 We apologize for any omission or error and thank in advance all readers that care to send us corrections.

3 To the best of our knowledge all Societies in the table were full members at the time of the published article. Some may have ceased to be so in the meantime.
} 
Societies

\begin{tabular}{|c|c|c|c|c|}
\hline \multirow{3}{*}{$\begin{array}{l}\text { Czech Mathematical Society } \\
\text { (Union of Czechs Mathematicians \& Physicists) }\end{array}$} & \multirow{3}{*}{$\begin{array}{l}\text { Czech } \\
\text { Republic }\end{array}$} & \multirow[t]{3}{*}{1862} & 1 & $09 / 1991$ \\
\hline & & & 43 & $03 / 2002$ \\
\hline & & & 85 & $09 / 2012$ \\
\hline \multirow[t]{2}{*}{ Danish Mathematical Society } & \multirow[t]{2}{*}{ Denmark } & \multirow[t]{2}{*}{1873} & 35 & $03 / 2000$ \\
\hline & & & 88 & $06 / 2013$ \\
\hline \multirow[t]{2}{*}{ Edinburgh Mathematical Society } & \multirow[t]{2}{*}{ UK } & \multirow[t]{2}{*}{1883} & 33 & $09 / 1999$ \\
\hline & & & 74 & $12 / 2009$ \\
\hline Estonian Mathematical Society & Estonia & 1926 & 41 & $09 / 2001$ \\
\hline \multirow[t]{2}{*}{ Finnish Mathematical Society } & \multirow[t]{2}{*}{ Finland } & \multirow[t]{2}{*}{1868} & 9 & $09 / 1993$ \\
\hline & & & 32 & $06 / 1999$ \\
\hline French Statistical Society & France & 1997 & & \\
\hline \multirow[t]{2}{*}{ German Mathematical Society } & \multirow[t]{2}{*}{ Germany } & \multirow[t]{2}{*}{1890} & 29 & $09 / 1998$ \\
\hline & & & 82 & $12 / 2011$ \\
\hline Georgian Mathematical Union & Georgia & 1923 & & \\
\hline Hellenic Mathematical Society & Greece & 1918 & 45 & $09 / 2002$ \\
\hline Icelandic Mathematical Society & Iceland & 1947 & & \\
\hline Institute of Mathematics and its Applications & UK & 1964 & 91 & $03 / 2014$ \\
\hline \multirow[t]{2}{*}{ Irish Mathematical Society } & \multirow[t]{2}{*}{ Ireland } & \multirow[t]{2}{*}{1976} & 2 & $12 / 1991$ \\
\hline & & & 94 & $12 / 2014$ \\
\hline Israel Mathematical Union & Israel & & 47 & $03 / 2003$ \\
\hline Italian Mathematical Union & Italy & 1922 & 38 & $12 / 2000$ \\
\hline Janos Bolyai Mathematical Society & Hungary & 1891 & 59 & $03 / 2006$ \\
\hline Kharkov Mathematical Society & Ukraine & 1879 & 34 & $12 / 1999$ \\
\hline Kosovar Mathematical Society & Kosovo & 2008 & & \\
\hline Latvian Mathematical Society & Latvia & 1993 & 48 & $06 / 2003$ \\
\hline Lithuanian Mathematical Society & Lithuania & 1962 & & \\
\hline \multirow[t]{4}{*}{ London Mathematical Society } & \multirow[t]{4}{*}{ UK } & 1865 & 31 & $03 / 1999$ \\
\hline & & & 37 & $09 / 2000$ \\
\hline & & & 99 & $03 / 2016$ \\
\hline & & & 103 & $03 / 2017$ \\
\hline Luxembourg Mathematical Society & Luxembourg & 1988 & 34 & $12 / 1999$ \\
\hline Malta Mathematical Society & Malta & & & \\
\hline Mathematical Society of France & France & 1872 & 40 & $06 / 2001$ \\
\hline Mathematical Society of Serbia & Serbia & 1948 & & \\
\hline Mathematical Society of the Republic of Moldova & Moldova & 1950s & & \\
\hline Moscow Mathematical Society & Russia & 1864 & 50 & $12 / 2003$ \\
\hline & & & 51 & $03 / 2004$ \\
\hline Norwegian Mathematical Society & Norway & 1918 & 41 & $09 / 2001$ \\
\hline Norwegian Statistical Association & Norway & 1919 & & \\
\hline Polish Mathematical Society & Poland & 1919 & 32 & $06 / 1999$ \\
\hline & & & 54 & $12 / 2004$ \\
\hline Portuguese Mathematical Society & Portugal & 1940 & 46 & $12 / 2002$ \\
\hline & & & 98 & $12 / 2015$ \\
\hline Romanian Mathematical Society & Romania & 1910 & 40 & $06 / 2001$ \\
\hline & & & 77 & $09 / 2010$ \\
\hline Royal Dutch Mathematical Society & Netherlands & 1778 & 35 & $03 / 2000$ \\
\hline Royal Spanish Mathematical Society & Spain & 1911 & & \\
\hline Slovak Mathematical Society & Slovakia & & & \\
\hline Società Italiana di Matematica Applicata e Industriale & Italy & 1989 & & \\
\hline Société de Mathématiques Appliquées et Industrielles & France & 1983 & 6 & $12 / 1992$ \\
\hline & & & 40 & $06 / 2001$ \\
\hline Society of Applied Mathematics and Mechanics & Germany & 1922 & & \\
\hline Society of Mathematicians and Physicists of Montenegro & Montenegro & 1947 & & \\
\hline
\end{tabular}


Mathematics Education

\begin{tabular}{|l|l|l|l|l|}
\hline $\begin{array}{l}\text { Society of Mathematicians, Physicists and Astronomers of } \\
\text { Slovenia }\end{array}$ & Slovenia & 1949 & 47 & $03 / 2003$ \\
\hline Spanish Society of Applied Mathematics & Spain & 1991 & & \\
\hline Spanish Society of Statistics and Operations Research & Spain & 1962 & & \\
\hline St. Petersburg Mathematical Society & Russia & 1890 & 10 & $12 / 1993$ \\
\hline Swedish Mathematical Society & Sweden & 1950 & & \\
\hline Swiss Mathematical Society & Switzerland & 1910 & 33 & $09 / 1999$ \\
\hline Turkish Mathematical Society & Turkey & 1948 & & \\
\hline Ukrainian Mathematical Society & Ukraine & & & \\
\hline Union of Bulgarian Mathematicians & Bulgaria & 1898 & 6 & $12 / 1992$ \\
\hline
\end{tabular}

